\title{
Low Serum Allopregnanolone Is Associated with Symptoms of Depression in Late Pregnancy
}

\author{
Charlotte Hellgren $^{a} \quad$ Helena Åkerud $^{a} \quad$ Alkistis Skalkidou ${ }^{a} \quad$ Torbjörn Bäckström ${ }^{b}$ \\ Inger Sundström-Poromaa ${ }^{a}$ \\ aDepartment of Women's and Children's Health, Uppsala University, Uppsala, and b Umeå Neurosteroid Research \\ Center, Department of Clinical Science, Obstetrics and Gynecology, Umeå University, Umeå, Sweden
}

\section{Key Words}

Allopregnanolone · Anxiety · Depression .

Montgomery-Åsberg Depression Rating Scale .

Pregnancy · Spielberger State-Trait Anxiety Inventory

\begin{abstract}
Background: Allopregnanolone (3a-hydroxy-5a-pregnan20-one) is a neurosteroid which has an inhibitory function through interaction with the $G A B A_{A}$ receptor. This progesterone metabolite has strong sedative and anxiolytic properties, and low endogenous levels have been associated with depressed mood. This study aimed to investigate whether the very high serum allopregnanolone levels in late pregnancy covary with concurrent self-rated symptoms of depression and anxiety. Methods: Ninety-six women in pregnancy weeks 37-40 rated symptoms of depression and anxiety with the Montgomery-Åsberg Depression Rating Scale (MADRS-S) and Spielberger State-Trait Anxiety Inventory. Their serum allopregnanolone was analyzed by Celite chromatography and radioimmunoassay. Results: Ten women had elevated depression scores (MADRS-S $\geq 13$ ), and this group had significantly lower allopregnanolone levels compared to women with MADRS-S scores in the normal range $(39.0 \pm 17.9$ vs. $54.6 \pm 18.7 \mathrm{nmol} / \mathrm{l}, \mathrm{p}=0.014)$. A significant negative correlation was found between self-rated depres-
\end{abstract}

sion scores and allopregnanolone concentrations (Pearson's correlation coefficient $=-0.220, p=0.031$ ). The linear association between self-rated depression scores and allopregnanolone serum concentrations remained significant when adjusted for gestational length, progesterone levels, and parity. Self-rated anxiety, however, was not associated with allopregnanolone serum concentrations during pregnancy. Conclusion: High allopregnanolone serum concentrations may protect against depressed mood during pregnancy.

(c) 2014 S. Karger AG, Basel

\section{Introduction}

Allopregnanolone ( $3 \alpha$-hydroxy-5a-pregnan-20-one) is a steroid which allosterically enhances GABAergic signaling at $\mathrm{GABA}_{\mathrm{A}}$ receptors [1]. Allopregnanolone is synthesized in the brain, but the main sources of serum allopregnanolone in nonpregnant women are the corpus luteum and the adrenal cortex $[2,3]$. During the men-

I. Sundström-Poromaa occasionally serves on advisory boards or acts as invited speaker at scientific meetings for MSD, Bayer Health Care, and Lundbeck $A / S$. The other authors declare that they have no conflicts of interest.

\begin{tabular}{ll}
\hline KARGER & $\begin{array}{l}\text { ○ 2014 S. Karger AG, Basel } \\
0302-282 X / 14 / 0693-0147 \$ 39.50 / 0 \quad \text { Karger }\end{array}$ \\
E-Mail karger@karger.com & $\begin{array}{l}\text { This is an Open Access article licensed under the terms of the } \\
\text { Creative Commons Attribution-NonCommercial 3.0 Un- } \\
\text { ported license (CC BY-NC) (www.karger.com/OA-license), } \\
\text { applicable to the online version of the article only. Distribu- } \\
\text { tion permitted for non-commercial purposes only. }\end{array}$
\end{tabular}

Charlotte Hellgren, $\mathrm{PhD}$

Department of Women's and Children's Health

Uppsala University

SE-751 85 Uppsala (Sweden)

E-Mail charlotte.hellgren@kbh.uu.se 
strual cycle, serum allopregnanolone concentrations vary between around $0.5 \mathrm{nmol} / \mathrm{l}$ in the follicular phase, and 4-5 nmol/l in the mid-luteal phase [2]. During pregnan$\mathrm{cy}$, fetoplacental synthesis causes the maternal serum concentrations of allopregnanolone to continuously rise to eventually reach more than 10 times the maximum menstrual cycle levels [4-6]. After delivery, the allopregnanolone level rapidly drops to $2 \mathrm{nmol} / \mathrm{l}$ within a few days, but slightly elevated levels (approximately $1 \mathrm{nmol} / \mathrm{l}$ ) are seen several weeks following parturition $[7,8]$. When intravenously administered to nonpregnant women, pregnancy-like allopregnanolone serum concentrations are sedative [9], and impair immediate recall [10], symptoms which do not affect late pregnant women in general [11]. These observations suggest that a tolerance to allopregnanolone develops during pregnancy.

Low levels of allopregnanolone have been implicated in mood disorder pathophysiology, for example, successful treatment with selective serotonin reuptake inhibitors increases plasma and cerebrospinal fluid allopregnanolone in patients with major depression $[12,13]$. However, increased serum allopregnanolone has also been observed after unsuccessful treatment with antidepressants [14], and no increase has been shown after successful electroconvulsive treatment [15]. Furthermore, altered neurosteroid metabolism has been observed in women with remitted depression compared to women with no history of depression [16]. Lower serum allopregnanolone in depressed compared to healthy menopausal women has also been reported [17]. Thus far, only one study has investigated the relationship between pregnancy allopregnanolone levels and depression, and found no difference in allopregnanolone levels between pregnant women with and without depression [8]. However, lower allopregnanolone levels after delivery have been found in women who develop postpartum blues [7], and for this reason, postpartum blues has been suggested to be an effect of withdrawal from allopregnanolone [18]. Hypothetically, women with higher allopregnanolone concentrations during pregnancy could be subjected to a more marked drop after delivery, and thereby be more susceptible to depressive symptoms postpartum, while they could be more protected from depression during pregnancy.

When administered acutely to female rats, allopregnanolone is known to be anxiolytic [19], whereas prolonged administration or withdrawal from allopregnanolone may precipitate increased anxiety [18]. Allopregnanolone is partly responsible for the decreased hypothalamic-pituitary-adrenal (HPA) response to stress seen in rats during pregnancy [20], and possibly also for the decreased anxiety behavior of pregnant rats [21]. Although the endocrine stress response is decreased also in human pregnancy, longitudinal data suggests that anxiety levels in healthy women are unchanged by pregnancy [22]. In addition, endogenous levels of allopregnanolone do not seem to be altered in anxiety disorders [23-26], other than panic disorder $[27,28]$.

The primary aim of this study was to assess self-rated symptoms of depression and anxiety in relation to serum levels of allopregnanolone in pregnant women. We hypothesized that higher levels of allopregnanolone during late pregnancy would be protective against concurrent symptoms of depressed mood and anxiety.

\section{Methods}

Subjects

Ninety-eight women in pregnancy weeks 37-40 were recruited through public maternity health care units in Uppsala County and through local newspaper advertisements. Women above 18 years of age with an uncomplicated singleton pregnancy were eligible for inclusion. Two women were excluded because of ongoing treatment with selective serotonin reuptake inhibitors, thus 96 women were included in the analyses.

The study procedures were in accordance with ethical standards for human experimentation and the study was approved by the Regional Ethical Review Board in Uppsala.

\section{Procedure}

The participants were scheduled to visit the Department of Women's and Children's Health, Uppsala University, between $08.00 \mathrm{~h}$ and $18.00 \mathrm{~h}$, approximately 2 weeks before their estimated date of delivery. Upon arrival, they provided written informed consent and had a blood sample taken. All women were asked about obstetric history, medication during the preceding 3 months, and tobacco and alcohol use. Ongoing depressive disorders and ongoing primary anxiety disorders were assessed with the Mini International Neuropsychiatric Interview, a structured interview based on DSM-IV criteria [29].

Subjects also filled out the Montgomery-Åsberg Depression Rating Scale (self-rated version, MADRS-S) [30] and the Spielberger State-Trait Anxiety Inventory (State and Trait versions, STAI-S and STAI-T, respectively) [31]. Although the MADRS-S is primarily designed to detect change, a score of 13 or more is commonly used in Swedish primary care to screen for ongoing depression, and was considered as an elevated depression score in this study. In the STAI-S and -T, a cut-off score of 40 gives around $80 \%$ sensitivity and specificity for anxiety disorders in a pregnant population [32].

First-trimester weight, date of delivery, and information on obstetric complications were extracted from hospital records.

All participants received the Swedish version of the Edinburgh Postnatal Depression Scale (EPDS) $[33,34]$ form to fill in and return by mail at six weeks after the delivery. As suggested by the Swedish EPDS validation [34], a score of 12 or more was considered screen-positive. 
Serum Steroid Concentration Analyses

A blood sample was collected by antecubital vein puncture and centrifuged at $1,500 \mathrm{~g}$ for $10 \mathrm{~min}$ and stored at $-20^{\circ} \mathrm{C}$ within an hour after sampling. The estradiol and progesterone analyses were carried out by competitive immunometric electrochemistry luminescence detection at the Department of Clinical Chemistry, Uppsala University Hospital. The samples were run on a Roche Cobas e601 with Cobas Elecsys estradiol and progesterone reagent kits, respectively (Roche Diagnostics, Bromma, Sweden). For progesterone the measurement interval was $0.1-191 \mathrm{nM}$ and for estradiol 18.4-15,781 pM. Progesterone intra-assay coefficient of variation was $2.2 \%$ at $2.39 \mathrm{nmol} / \mathrm{l}$ and $2.8 \%$ at $31.56 \mathrm{nmol} / \mathrm{l}$. The total coefficient of variation was $4.8 \%$ at $2.52 \mathrm{nmol} / \mathrm{l}$ and $2.0 \%$ at $112 \mathrm{nmol} / \mathrm{l}$. Estradiol intra-assay coefficient of variation was $6.8 \%$ at $85.5 \mathrm{pmol} / \mathrm{l}$ and $2.8 \%$ at $1,640 \mathrm{pmol} / \mathrm{l}$. The total coefficient of variation was $4.7 \%$ at $120 \mathrm{pmol} / \mathrm{l}$ and $2.6 \%$ at $12,935 \mathrm{pmol} / \mathrm{l}$.

Allopregnanolone serum concentrations were determined by Umeå Neurosteroid Research Center, as previously described [9]. Briefly, serum allopregnanolone was measured using radioimmunoassay after extraction with diethyl ether and purification by Celite chromatography because of the cross-reactivity of the antibody. The antibody used was raised against $3 a$-hydroxy-20-oxo5a-pregnan-11-yl carboxymethyl ether coupled with bovine serum albumin as antigen (AgriSera AB, Umeå, Sweden). All samples were counted in a RackBeta (Wallace, Finland) scintillation counter.

Statistical Analyses

With a standard deviation (SD) of $14 \mathrm{nM}$ [5] we had a power of 0.83 to detect a mean difference of $15 \mathrm{~nm}$ between the women with depression scores of 13 or more and women with depression scores in the normal range.

Allopregnanolone and estradiol concentrations, trait anxiety scores, and days to delivery were found to be normally distributed (Shapiro-Wilk, W > 0.95). However, the depression scores (MADRS-S and EPDS), progesterone concentrations, state anxiety scores, and weight were not considered normally distributed (Shapiro-Wilk, $\mathrm{W}<0.95)$, but Pearson's correlations were calculated since the unstandardized residuals of each presented univariate linear correlation were found to follow normal distribution. However, potential differences in hormonal and demographic variables between women with MADRS-S scores $\geq 13$ and $<13$ were assessed with the Mann-Whitney U test, or Fisher's exact test.

The association between allopregnanolone and depression scores, adjusted for potential confounders, was tested with linear regression.

SPSS Statistics 20 (IBM) was used in all statistical analyses; $p$ values $<0.05$ were considered statistically significant and data is presented as mean \pm SD unless stated otherwise.

\section{Results}

\section{Demographic Factors and Obstetric Outcome}

The mean age of the women was $30.7 \pm 4.5$ years and 55 (57.3\%) were nulliparous (pregnant with their first child). The assessments were made in gestational week $38.2 \pm 0.6$ and the median number of days until parturition was 13.5 days (range 1-32 days). All but 1 woman were married or
Table 1. Study population characteristics

\begin{tabular}{|c|c|}
\hline & Mean \pm SD \\
\hline First-trimester weight, kg & $69.6 \pm 14.0$ \\
\hline Late pregnancy serum progesterone, $\mathrm{nmol} / \mathrm{l}$ & $683 \pm 229$ \\
\hline Late pregnancy serum estradiol ${ }^{\mathrm{a}}, \mathrm{nmol} / \mathrm{l}$ & $89.3 \pm 33.3$ \\
\hline Late pregnancy serum allopregnanolone, $\mathrm{nmol} / \mathrm{l}$ & $53.0 \pm 19.1$ \\
\hline Late pregnancy STAI-S score & $30.2 \pm 6.5$ \\
\hline Late pregnancy STAI-T score & $32.4 \pm 6.3$ \\
\hline Late pregnancy MADRS-S score & $6.4 \pm 4.3$ \\
\hline Postpartum EPDS score ${ }^{\mathrm{b}}$ & $4.9 \pm 3.8$ \\
\hline
\end{tabular}

\footnotetext{
${ }^{\text {a }}$ Estradiol was determined in 95 women.

${ }^{\mathrm{b}}$ EPDS forms were received from 90 women.
}

cohabiting. No women reported use of alcohol during pregnancy, but 1 woman reported tobacco use. Eight women $(8.3 \%)$ were delivered by Caesarean section and the remaining women had a vaginal delivery. None of the women experienced any severe delivery complications.

\section{Depression and Anxiety during Pregnancy}

Ten women (10.4\%) had MADRS-S scores $\geq 13$, indicating ongoing depressive symptoms. According to the Mini International Neuropsychiatric Interview, 2 women (2.1\%) had an ongoing major depressive episode, 1 woman (1.0\%) had obsessive compulsive disorder, and 1 woman $(1.0 \%)$ was found to suffer from recurrent panic attacks but without fulfilling the criteria for panic disorder; these 4 women also had MADRS-S scores $\geq 13$. Additional study population characteristics including mean MADRS-S and STAI and steroid hormone concentrations are found in table 1.

\section{Steroid Concentrations in Relation to Depression and} Anxiety Scores during Pregnancy

Clinical characteristics and hormonal serum concentrations in women who had elevated or normal range depression scores are given in table 2 . Women with elevated depression scores had significantly lower allopregnanolone serum concentrations than women with depression scores in the normal range [medians (interquartile range) $36.9(23.8-50.1)$ vs. $51.8(40.7-67.3) \mathrm{nmol} / \mathrm{l}, \mathrm{p}=0.014$, table 2]. The 4 women who had an anxiety or depressive disorder according to Mini International Neuropsychiatric Interview had a mean allopregnanolone plasma concentration of $27.2 \pm 10.3 \mathrm{nmol} / \mathrm{l}$.

Allopregnanolone serum concentrations were in statistically significant negative correlation with self-report- 
Table 2. Demographic data and hormone levels in women with MADRS-S scores $<13$ or $\geq 13$, presented as median (interquartile range)

\begin{tabular}{lccc}
\hline & $\begin{array}{l}\text { MADRS-S scores }<13 \\
(\mathrm{n}=86)\end{array}$ & $\begin{array}{l}\text { MADRS-S scores } \geq 13 \\
(\mathrm{n}=10)\end{array}$ & $\mathrm{p}^{\text {value }}$ \\
\hline Age, years & $30.5(27.8-33.0)$ & $30.0(26.8-35.8)$ & 0.904 \\
Nulliparous, $\mathrm{n}(\%)$ & $52(60.5)$ & $3(30.0)$ & 0.092 \\
First-trimester weight, kg & $67.8(61.0-72.0)$ & $64.0(58.8-85.6)$ & 0.895 \\
Days left to delivery, $\mathrm{n}$ & $14(8.8-20.3)$ & $12.5(8.0-18.0)$ & 0.442 \\
Serum allopregnanolone, nmol/1 & $51.8(40.7-67.3)$ & $36.9(23.8-50.1)$ & 0.014 \\
Serum progesterone, nmol/l & $642(525-797)$ & $679(455-792)$ & 0.919 \\
Serum estradiol, nmol/l & $83.2(65.8-114.9)$ & $82.9(71.1-89.2)$ & 0.536 \\
\hline
\end{tabular}

${ }^{a}$ Mann-Whitney U test or Fisher's exact test was used for groupwise comparisons.

Table 3. Pearson correlation coefficients for correlations between allopregnanolone serum concentrations and hormonal, gestational and mood variables

\begin{tabular}{|c|c|c|c|c|c|c|}
\hline & Progesterone & Estradiol & Days before delivery & MADRS-S & STAI-S & STAI-T \\
\hline Allopregnanolone & $\begin{array}{l}0.530 \\
p<0.001\end{array}$ & $\begin{array}{l}0.086 \\
p=0.406\end{array}$ & $\begin{array}{l}-0.247 \\
\mathrm{p}=0.015\end{array}$ & $\begin{array}{l}-0.220 \\
p=0.031\end{array}$ & $\begin{array}{l}0.031 \\
p=0.767\end{array}$ & $\begin{array}{l}0.027 \\
p=0.794\end{array}$ \\
\hline
\end{tabular}

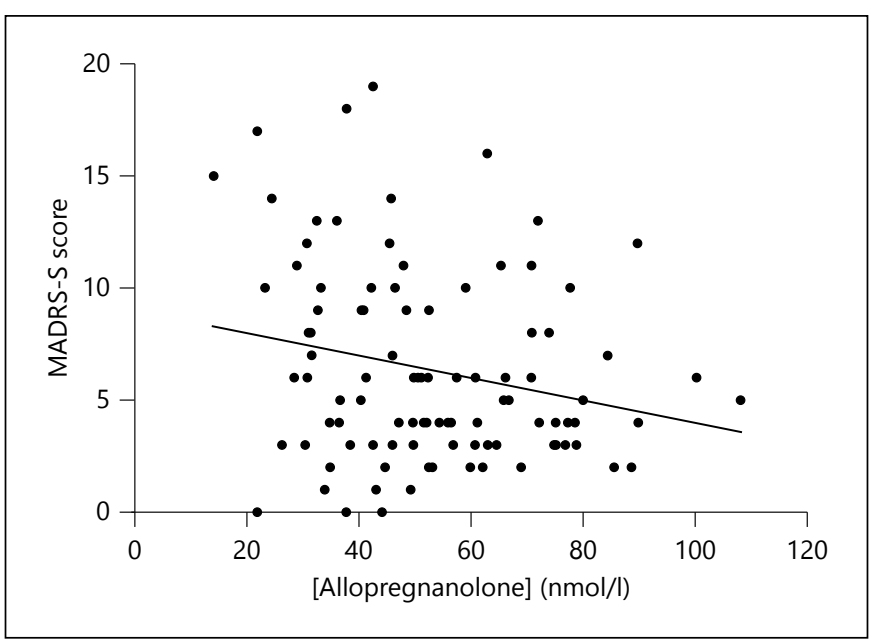

Fig. 1. Linear regression of MADRS-S score during weeks 36-40 of pregnancy as a function of allopregnanolone serum concentration $(\mathrm{r}=-0.220, \mathrm{p}=0.031)$.

ed depression scores (Pearson's correlation coefficient $=$ $-0.220, \mathrm{p}=0.031$; fig. 1 ).

Neither progesterone nor estradiol concentrations were associated with pregnancy depression scores (both $\mathrm{p}>0.20)$. State and trait anxiety ratings were not corre- lated with allopregnanolone, estradiol, or progesterone concentrations (all p > 0.20).

Allopregnanolone was also associated with the number of days left to delivery, and serum progesterone (table 3). There was no effect of time of blood sampling on the allopregnanolone concentration (a.m. vs. p.m. samples, $\mathrm{p}=0.213$ ).

A greater proportion of women with elevated depression scores had previously given birth, although this difference was not significant ( $p=0.092$, Fisher's exact test). No other significant differences in age, first-trimester weight, progesterone, or estradiol levels were found between women with or without elevated depression scores.

The correlation between allopregnanolone and depression score remained after adjustment for days left to delivery, serum progesterone, and nulliparity (table 4).

\section{Postpartum Depression Scores}

Ninety women $(93.8 \%)$ sent in their EPDS forms at $6.9 \pm 1.8$ weeks postpartum. Seven of them $(7.8 \%)$ had a score of $\geq 12$, one of whom had elevated depression scores also during late pregnancy. However, 4 of the women who had pregnancy MADRS-S scores $\geq 13$ did not return their postpartum EPDS forms. There was no association between postpartum depression scores and allopreg- 
Table 4. Linear regression of explanatory variables for MADRS-S score during pregnancy

\begin{tabular}{lcl}
\hline Variables in model & Linear model $\beta(95 \%)$ & p value \\
\hline $\begin{array}{l}\text { Unadjusted } \\
\text { Serum allopregnanolone }\end{array}$ & $-0.050(-0.095$ to -0.005$)$ & 0.031 \\
\hline Adjusted & & \\
Serum allopregnanolone & $-0.056(-0.111$ to -0.001$)$ & 0.048 \\
Days to delivery & $-0.022(-0.140$ to -0.095$)$ & 0.707 \\
Serum progesterone & $0.001(-0.004$ to 0.005$)$ & 0.796 \\
Nulliparity & $-0.177(-1.594$ to 1.949$)$ & 0.843 \\
\hline
\end{tabular}

nanolone concentrations during pregnancy (Pearson correlation $0.047, \mathrm{p}=0.658$ ), and thus we did not proceed with regression analysis.

\section{Discussion}

We observed a negative association between late pregnancy serum allopregnanolone and self-rated depression scores. This association was driven by the women who had elevated depression scores, who displayed significantly lower allopregnanolone serum concentrations in comparison with women with scores in the normal range.

In disagreement with our results, one previous study examining clinically depressed and euthymic pregnant women found no difference in allopregnanolone levels [8]. One explanation for the discrepant findings could be that the group sizes of Pearson Murphy et al. [8] were too small to detect the relatively modest effect of allopregnanolone suggested by our results. In addition, Pearson Murphy et al. [8] studied a population of women with first onset of clinical depression during pregnancy; our subjects represent a relatively healthy pregnant population with varying degrees of depressed mood, in most cases outside the range of clinical disease. However, the allopregnanolone precursor $5 a$-dihydroprogesterone was elevated in the depressed women in the study by Pearson Murphy et al. [8], indicating a related neurosteroid imbalance. While the conflicting results underline the lack of direct clinical value of allopregnanolone during pregnancy, together they provide evidence for the importance of a general neurosteroid balance. Pregnancy and the postpartum period entail large neuroendocrine changes, and often increased stress. It is possible that the elevation of neuroactive progesterone metabolites, with their inhibitory potential, is one of the factors which ensure that most women maintain good

Allopregnanolone and Antenatal

Depressive Symptoms mental health throughout these challenges $[7,8]$, but that it is of less relevance for the development of clinical depression during pregnancy [8]. However, the lack of $5 a$-dihydroprogesterone analysis in the current study is a limitation to further elucidation of this idea.

Decreased allopregnanolone levels are found in major unipolar depression in humans $[12,13]$ and are related to anxiety- and depression-like behaviors in rodent models of mood disorders [35-37]. Low concentrations of allopregnanolone also correspond with anxiety levels and altered $\mathrm{GABA}_{\mathrm{A}}$ receptor function across the rodent estrous cycle $[38,39]$. However, the lowest allopregnanolone level within our study population was more than 7 times higher than typical maximum menstrual cycle levels [40], therefore no participant can be said to have had a low allopregnanolone serum concentration. It is also obvious that depression and anxiety are at least as common during pregnancy as during other time points in a woman's fertile life [41], despite the anxiolytic and sedative levels of allopregnanolone. Given the possibility that a tolerance develops during several months of continuous increase [42], it is reasonable that only women with very high levels, or a steeper increase, would benefit from the protective effects of allopregnanolone during pregnancy. Insensitivity to other substances acting on the $\mathrm{GABA}_{\mathrm{A}}$ receptor, as well as tolerance to the anticonvulsive, learning-impairing, and anesthetic effects of allopregnanolone exposure have been demonstrated in rodent models [42]. The $\mathrm{GABA}_{\mathrm{A}}$ receptor sensitivity to allopregnanolone may depend on receptor subunit composition, where the relative contributions of subunits $\alpha 4$ and $\delta$ have received most attention $[43,44]$. It is thus possible that altered subunit composition induces tolerance development during pregnancy, although it remains to be proven in humans.

Evidently, serum concentration is a very blunt measure when studying the effect of allopregnanolone on mood, which is most likely determined by local changes in neurosteroid synthesis and $\mathrm{GABA}_{\mathrm{A}}$ receptor subunit composition within the brain [43]. However, the association we observed suggests that the massive increase in peripheral fetoplacental allopregnanolone synthesis has the potential to affect the central nervous system and protect some women from negative mood symptoms in late pregnancy. Allopregnanolone readily crosses the blood-brain barrier, therefore the serum levels of the steroid are correlated with brain tissue levels [45]. In addition, allopregnanolone is accumulated in adipose tissue and in certain brain areas in rats [44].

In nonpregnant women, allopregnanolone release is coupled to HPA-axis activity [46]. Allopregnanolone decreases the HPA response in pregnant rodents [20] and 
in addition to the direct GABA-enhancing effect, allopregnanolone administration affects the transcription of genes involved in HPA-axis regulation in rats [47]. Thus, there is a possibility that the association seen in our study is mediated by HPA-axis regulation effects on mood [48].

Based on rodent studies [19,21], we had expected that high serum allopregnanolone would be a protective factor also against symptoms of anxiety. We found no such effect, perhaps because of our relatively nonanxious study population. It must also be mentioned that we measured state anxiety (referring to the momentary anxiety at the visit), and trait anxiety (referring to anxiety-prone personality), with the STAI inventory, which may have targeted negative affect more than symptoms of anxiety [49].

In addition, no association of allopregnanolone to postpartum depressive symptoms was found. Nappi et al. [7] have indicated a role for allopregnanolone withdrawal in the development of postpartum blues by reporting a negative correlation between clinician-rated depression scores and serum allopregnanolone in women 3 days after delivery. While their correlation is in agreement with our results during pregnancy, we could not show that a larger withdrawal, as approximated by higher pregnancy allopregnanolone, would increase depression scores also at 6-8 weeks postpartum. This can be due to the positive attrition in our postpartum depression score measurement, or to the fact that different rating scales were used on the two occasions, but it is also likely that allopregnanolone withdrawal has a larger influence during the first days following delivery than several weeks into the postpartum period. Clearly, this remains speculative due to our cross-sectional allopregnanolone analysis and further longitudinal studies are needed.

An important limitation to our study is the small number of women with symptoms of depression, which opens up for the possibility of a type I error. A $10 \%$ point prevalence of self-rated symptom scores indicating depression in late pregnancy is comparable with population-based studies [50,51], but future studies with larger numbers of women suffering from depressed mood are needed to prove the validity of the association with serum allopregnanolone.

In conclusion, the observed association between allopregnanolone and self-rated depression score indicates a role for allopregnanolone in mood regulation also during pregnancy. Although our findings may not be generalized to pregnant women suffering from major depression, the results are relevant for generally healthy pregnant women who experience mood symptoms during pregnancy.

\section{Acknowledgments}

The authors would like to sincerely thank the women who participated in this study. The authors would also like to express their gratitude to Lena Moby for valuable help during the study. In addition, Lisa Wernroth, and Liisa Byberg from Uppsala Clinical Research Center are acknowledged for helpful statistical advice.

The study was supported by research grants from the Swedish Research Council, the Council for Working Life and Social Research, and the Marianne and Marcus Wallenberg Foundation. None of the funding sources have influenced the study design, data collection, data analysis, manuscript preparation, or submission process.

\section{References}

$\checkmark 1$ Majewska MD, Harrison NL, Schwartz RD, Barker JL, Paul SM: Steroid hormone metabolites are barbiturate-like modulators of the GABA receptor. Science 1986;232:10041007.

2 Genazzani AR, Petraglia F, Bernardi F, Casarosa E, Salvestroni $\mathrm{C}$, Tonetti A, et al: Circulating levels of allopregnanolone in humans: gender, age, and endocrine influences. J Clin Endocrinol Metab 1998;83:2099-2103.

-3 Ottander U, Poromaa IS, Bjurulf E, Skytt A, Backstrom T, Olofsson JI: Allopregnanolone and pregnanolone are produced by the human corpus luteum. Mol Cell Endocrinol 2005;239:37-44.

$\checkmark 4$ Dombroski RA, Casey ML, MacDonald PC: 5-Alpha-dihydroprogesterone formation in human placenta from 5alpha-pregnan-3beta/ alpha-ol-20-ones and 5-pregnan-3beta-yl20-one sulfate. J Steroid Biochem Mol Biol 1997;63:155-163.
5 Luisi S, Petraglia F, Benedetto C, Nappi RE, Bernardi F, Fadalti M, et al: Serum allopregnanolone levels in pregnant women: changes during pregnancy, at delivery, and in hypertensive patients. J Clin Endocrinol Metab 2000;85:2429-2433.

6 Parizek A, Hill M, Kancheva R, Havlikova H, Kancheva L, Cindr J, et al: Neuroactive pregnanolone isomers during pregnancy. J Clin Endocrinol Metab 2005;90:395-403.

7 Nappi RE, Petraglia F, Luisi S, Polatti F, Farina C, Genazzani AR: Serum allopregnanolone in women with postpartum 'blues'. Obstet Gynecol 2001;97:77-80.

8 Pearson Murphy BE, Steinberg SI, Hu FY, Allison CM: Neuroactive ring A-reduced metabolites of progesterone in human plasma during pregnancy: elevated levels of 5 alphadihydroprogesterone in depressed patients during the latter half of pregnancy. J Clin Endocrinol Metab 2001;86:5981-5987.
9 Timby E, Balgard M, Nyberg S, Spigset O, Andersson A, Porankiewicz-Asplund J, et al: Pharmacokinetic and behavioral effects of allopregnanolone in healthy women. Psychopharmacology (Berl) 2006;186:414424

10 Kask K, Bäckström T, Nilsson LG, Sundström-Poromaa I: Allopregnanolone impairs episodic memory in healthy women. Psychopharmacology (Berl) 2008;199:161168.

11 Christensen H, Leach LS, Mackinnon A: Cognition in pregnancy and motherhood: prospective cohort study. Br J Psychiatry 2010; 196:126-132

12 Romeo E, Strohle A, Spalletta G, di Michele F, Hermann B, Holsboer F, et al: Effects of antidepressant treatment on neuroactive steroids in major depression. Am J Psychiatry 1998; 155:910-913. 
13 Uzunova V, Sheline Y, Davis JM, Rasmusson 25 Le Melledo JM, Van Driel M, Coupland NJ, A, Uzunov DP, Costa E, et al: Increase in the cerebrospinal fluid content of neurosteroids in patients with unipolar major depression who are receiving fluoxetine or fluvoxamine. Proc Natl Acad Sci USA 1998;95:3239-3244.

$\checkmark 14$ Schule C, Romeo E, Uzunov DP, Eser D, di Michele F, Baghai TC, et al: Influence of mirtazapine on plasma concentrations of neuroactive steroids in major depression and on 3alpha-hydroxysteroid dehydrogenase activity. Mol Psychiatry 2006;11:261-272.

15 Baghai TC, di Michele F, Schule C, Eser D, Zwanzger P, Pasini A, et al: Plasma concentrations of neuroactive steroids before and after electroconvulsive therapy in major depression. Neuropsychopharmacology 2005;30: 1181-1186.

16 Klatzkin RR, Morrow AL, Light KC, Pedersen CA, Girdler SS: Associations of histories of depression and PMDD diagnosis with allopregnanolone concentrations following the oral administration of micronized progesterone. Psychoneuroendocrinology 2006;31:1208-1219.

$\checkmark 17$ Morgan ML, Rapkin AJ, Biggio G, Serra M, Pisu MG, Rasgon N: Neuroactive steroids after estrogen exposure in depressed postmenopausal women treated with sertraline and asymptomatic postmenopausal women. Arch Womens Ment Health 2010;13:91-98.

18 Gulinello M, Orman R, Smith SS: Sex differences in anxiety, sensorimotor gating and expression of the alpha4 subunit of the GABAA receptor in the amygdala after progesterone withdrawal. Eur J Neurosci 2003;17:641-648.

19 Bitran D, Hilvers RJ, Kellogg CK: Anxiolytic effects of 3 alpha-hydroxy-5 alpha[beta]pregnan-20-one: endogenous metabolites of progesterone that are active at the GABAA receptor. Brain Res 1991;561:157-161.

$\checkmark 20$ Brunton PJ, McKay AJ, Ochedalski T, Piastowska A, Rebas E, Lachowicz A, et al: Central opioid inhibition of neuroendocrine stress responses in pregnancy in the rat is induced by the neurosteroid allopregnanolone. J Neurosci 2009;29:6449-6460.

21 de Brito Faturi C, Teixeira-Silva F, Leite JR: The anxiolytic effect of pregnancy in rats is reversed by finasteride. Pharmacol Biochem Behav 2006;85:569-574.

22 Paoletti AM, Romagnino S, Contu R, Orru MM, Marotto MF, Zedda P, et al: Observational study on the stability of the psychological status during normal pregnancy and increased blood levels of neuroactive steroids with GABA-A receptor agonist activity. Psychoneuroendocrinology 2006;31:485-492.

-23 Bicikova M, Tallova J, Hill M, Krausova Z, Hampl R: Serum concentrations of some neuroactive steroids in women suffering from mixed anxiety-depressive disorder. Neurochem Res 2000;25:1623-1627.

-24 Heydari B, Le Melledo JM: Low pregnenolone sulphate plasma concentrations in patients with generalized social phobia. Psychol Med 2002;32:929-933. Lott P, Jhangri GS: Response to flumazenil in women with premenstrual dysphoric disorder. Am J Psychiatry 2000;157:821-823.

26 Semeniuk T, Jhangri GS, Le Melledo JM: Neuroactive steroid levels in patients with generalized anxiety disorder. J Neuropsychiatry Clin Neurosci 2001;13:396-398.

27 Brambilla F, Biggio G, Pisu MG, Bellodi L, Perna G, Bogdanovich-Djukic V, et al: Neurosteroid secretion in panic disorder. Psychiatry Res 2003;118:107-116.

28 Strohle A, Romeo E, di Michele F, Pasini A, Yassouridis A, Holsboer F, et al: GABA(A) receptor-modulating neuroactive steroid composition in patients with panic disorder before and during paroxetine treatment. Am J Psychiatry 2002;159:145-147.

29 Sheehan DV, Lecrubier Y, Sheehan KH, Amorim P, Janavs J, Weiller E, et al: The Mini-International Neuropsychiatric Interview (M.I.N.I.): the development and validation of a structured diagnostic psychiatric interview for DSM-IV and ICD-10. J Clin Psychiatry 1998;59(suppl 20):22-33, quiz 34-57.

30 Svanborg P, Asberg M: A new self-rating scale for depression and anxiety states based on the Comprehensive Psychopathological Rating Scale. Acta Psychiatr Scand 1994;89:21-28.

31 Spielberger CD: State-Trait Anxiety Inventory for Adults. Sampler Set, Manual Set, Scoring Key. Palo Alto, Consulting Psychologists Press Inc, 1983.

32 Grant KA, McMahon C, Austin MP: Maternal anxiety during the transition to parenthood: a prospective study. J Affect Disord

33 Cox JL, Holden JM, Sagovsky R: Detection of postnatal depression: development of the 10item Edinburgh Postnatal Depression Scale. Br J Psychiatry 1987;150:782-786.

34 Wickberg B, Hwang CP: The Edinburgh Postnatal Depression Scale: validation on a Swedish community sample. Acta Psychiatr Scand 1996;94:181-184.

-35 Dong E, Matsumoto K, Uzunova V, Sugaya I, Takahata H, Nomura H, et al: Brain 5alphadihydroprogesterone and allopregnanolone synthesis in a mouse model of protracted social isolation. Proc Natl Acad Sci USA 2001; 98:2849-2854.

-36 Serra M, Pisu MG, Littera M, Papi G, Sanna $\mathrm{E}$, Tuveri $\mathrm{F}$, et al: Social isolation-induced decreases in both the abundance of neuroactive steroids and $\mathrm{GABA}(\mathrm{A})$ receptor function in rat brain. J Neurochem 2000;75:732-740.

- 37 Steimer T, Driscoll P, Schulz PE: Brain metabolism of progesterone, coping behaviour and emotional reactivity in male rats from two psychogenetically selected lines. J Neuroendocrinol 1997;9:169-175.

38 Finn DA, Gee KW: The influence of estrus cycle on neurosteroid potency at the gammaaminobutyric acidA receptor complex. J Pharmacol Exp Ther 1993;265:1374-1379. 2008; 108:101-111.
39 Frye CA, Petralia SM, Rhodes ME: Estrous cycle and sex differences in performance on anxiety tasks coincide with increases in hippocampal progesterone and 3alpha,5alpha-THP. Pharmacol Biochem Behav 2000;67:587-596.

40 Nyberg S, Bäckström T, Zingmark E, Purdy RH, Poromaa IS: Allopregnanolone decrease with symptom improvement during placebo and gonadotropin-releasing hormone agonist treatment in women with severe premenstrual syndrome. Gynecol Endocrinol 2007;23:257266.

41 Vesga-Lopez O, Blanco C, Keyes K, Olfson M, Grant BF, Hasin DS: Psychiatric disorders in pregnant and postpartum women in the United States. Arch Gen Psychiatry 2008;65:805-815.

42 Turkmen S, Backstrom T, Wahlstrom G, Andreen L, Johansson IM: Tolerance to allopregnanolone with focus on the GABA-A receptor. Br J Pharmacol 2011;162:311-327.

43 Smith SS, Shen H, Gong QH, Zhou X: Neurosteroid regulation of $\mathrm{GABA}(\mathrm{A})$ receptors: focus on the alpha 4 and delta subunits. Pharmacol Ther 2007;116:58-76.

44 Zhu D, Birzniece V, Bäckström T, Wahlström G: Dynamic aspects of acute tolerance to allopregnanolone evaluated using anaesthesia threshold in male rats. Br J Anaesth 2004;93:560-567.

45 Bixo M, Andersson A, Winblad B, Purdy RH, Bäckström T: Progesterone, 5alpha-pregnane-3,20-dione and 3alpha-hydroxy-5alpha-pregnane-20-one in specific regions of the human female brain in different endocrine states. Brain Res 1997;764:173-178.

46 Genazzani AD, Luisi M, Malavasi B, Strucchi C, Luisi S, Casarosa E, et al: Pulsatile secretory characteristics of allopregnanolone, a neuroactive steroid, during the menstrual cycle and in amenorrheic subjects. Eur J Endocrinol 2002;146:347-356.

47 Patchev VK, Hassan AH, Holsboer DF, Almeida OF: The neurosteroid tetrahydroprogesterone attenuates the endocrine response to stress and exerts glucocorticoid-like effects on vasopressin gene transcription in the rat hypothalamus. Neuropsychopharmacology 1996;15:533-540.

48 Rich-Edwards JW, Mohllajee AP, Kleinman K, Hacker MR, Majzoub J, Wright RJ, et al: Elevated midpregnancy corticotropin-releasing hormone is associated with prenatal, but not postpartum, maternal depression. J Clin Endocrinol Metab 2008;93:1946-1951.

49 Bados A, Gomez-Benito J, Balaguer G: The state-trait anxiety inventory, trait version: does it really measure anxiety? J Pers Assess 2010;92:560-567.

50 Andersson L, Sundström-Poromaa I, Wulff M, Aström M, Bixo M: Depression and anxiety during pregnancy and six months postpartum: a follow-up study. Acta Obstet Gynecol Scand 2006;85:937-944.

51 Heron J, O’Connor TG, Evans J, Golding J, Glover V: The course of anxiety and depression through pregnancy and the postpartum in a community sample. J Affect Disord 2004;80: 65-73.
Allopregnanolone and Antenatal

Depressive Symptoms
Neuropsychobiology 2014;69:147-153 DOI: $10.1159 / 000358838$ 